\title{
A revised multidimensional social desirability inventory
}

\author{
LEONARD J. JACOBSON, RICHARD F. BROWN, and MARIA J. ARIZA \\ University of Miami, Coral Gables, Florida
}

\begin{abstract}
A multidimensional social desirability inventory was revised in order to facilitate the usefulness and improve the precision of measurement of its four subscales. The original inventory was based on a conception of the importance of attribution and denial as central and quantifiable elements in self-descriptive statements differing in social desirability value. The 442 subjects participated in a series of investigations that resulted in the creation of a 128-item scale with 32 items in each of the following categories: attribution of positive traits, attribution of negative traits, denial of positive traits, and denial of negative traits. The importance of providing operational definitions for the measurement and quantification of sources of variation in self-descriptive statements was discussed.
\end{abstract}

In a previous study, Jacobson, Kellogg, Cauce, and Slavin (1977) conceptualized the need for social approval as a multidimensional construct consisting of at least partially independent dimensions. Based on this theoretical model, the Jacobson-Kellogg Self-Description Inventory (SDI-I) was created. The inventory consists of four subscales that measure the frequency of endorsement of statements of each of the following: the attribution of positive traits, the attribution of negative traits, the denial of positive traits, and the denial of negative traits. The inventory was designed also to control for the possible operation of both agreement and acceptance acquiescence response sets (Bentler, Jackson, \& Messick, 1971). The data presented by Jacobson et al., as well as by subsequent studies (Ariza, 1982; Millham \& Jacobson, 1978; Millham \& Kellogg, 1980; Ramanaiah, Schill, \& Leung, 1977), have continued to provide support for the conceptualization of social desirability as a multidimensional construct.

One difficulty with the SDI-I was that each subscale comprised only 17 items. The limited number of items prevented investigators from making full use of the scale through employment of the conventional dichotomous and trichotomous divisions (e.g., high, medium, low) frequent in personality and social research. Therefore, investigators have used only the total score and have avoided use of the subscales. Although the total score is certainly the single most useful indicator of performance, the purpose of having four separate subscales was to encourage their specific use for research purposes. Therefore, the present revision of the scale was undertaken in order to increase the number of items within each subscale and thereby to facilitate the subscale's use in research.

Copies of the scale may be obtained by writing to: Leonard I. Jacobson, Department of Psychology, University of Miami, Coral Gables, Florida 33124.

\section{METHOD}

\section{Subjects}

The subjects were 442 male and female undergraduate students enrolled in introductory psychology courses at the University of Miami.

\section{The Jacobson-Kellogg Self-Description Inventory-Revised Version (SDI-II)}

As in the original inventory, four scales make up the SDI-II. Specifically, they provide operational definitions of the extent to which subjects accept or deny traits differing in social desirability value (i.e., attribution of positive traits, attribution of negative traits, denial of positive traits, and denial of negative traits)

Subscale 1-Attribution of positive traits. This subscale comprises items for which the socially desirable response is "true." A true response is one in which the subject attributes to the self a socially desirable stem behavior: "I always keep my promises," or "I obey all traffic laws."

Subscale 2-Attribution of negative traits. This subscale comprises items for which the socially desirable response is "false." A true response is one in which the subject attributes to the self a socially undesirable stem behavior: "I sometimes try to dodge responsibilities," or "I sometimes lie to make a better impression."

Subscale 3-Denial of positive traits. This subscale comprises items for which the socially desirable response is "false." A true response is one in which the subject denies a socially desirable stem behavior: "I am not always a good listener," or "I do not wake up fresh and rested every morning."

Subscale 4-Denial of negative traits. This subscale comprises items for which the socially desirable response is "true." A true response is one in which the subject denies a socially undesirable stem behavior: "I never blame others for my own mistakes," or "I never lie to get out of work."

\section{Inventory Construction}

A pool of 350 items, including all of the items found in the original scale, was created. Of these, the investigators agreed that 268 had good content validity. The items were administered to 133 subjects, with 67 items for each subscale. Point biserial correlations of items and total subscale scores were computed, and those items that failed to meet the .01 level of significance were eliminated. Of the 268 items, 173 met criterion and were administered to another 89 subjects. This time 136 items met criterion. 
Table 1

Intercorrelations Among the SDI-II Subscales

\begin{tabular}{lcccc} 
& \multicolumn{5}{c}{ Subscale } \\
\cline { 2 - 5 } Subscale & 1 & 2 & 3 & 4 \\
\hline 2 & .673 & & & \\
3 & .569 & .615 & & \\
4 & .776 & .774 & .600 & \\
Total & .882 & .895 & .735 & .914 \\
\hline
\end{tabular}

Note $-N=108 ;$ all ps $<.001$.

Table 2

Means, Standard Deviations (SD), and Reliability Coefficients (R) of the Measures Employed

\begin{tabular}{cccc}
\hline Subscale & Mean & SD & R \\
\hline 1 & 17.85 & 6.98 & .88 \\
2 & 16.11 & 7.16 & .89 \\
3 & 17.27 & 5.79 & .81 \\
4 & 13.24 & 7.06 & .89 \\
Total & 64.67 & 24.07 & .96 \\
\hline
\end{tabular}

Note $-N=108$.

These items were administered to a sample of 112 subjects; all of the items reached significance or were marginally close to it. These borderline items were deleted, and a final scale of 128 items remained, all of which met criterion for a sample of 108 subjects. The final scale consisted of 128 items $(32$ items per subscale) that survived this rigorous cross-validation.

\section{RESULTS AND DISCUSSION}

The intercorrelations among the scales of the SDI-II are presented in Table 1. The table indicates a pattern of moderate correlations among the subscales and a high correlation of each with total score. These results are similar to those found in the original study. The means, standard deviations, and reliability coefficients are presented in Table 2. It may be noted that the reliability
(Kuder-Richardson Formula 20) of the subscales and the total score was uniformly high. Analysis of variance confirmed our previous findings that no effects of sex are in evidence for any subscale or for total score.

As a result of this revision, it is now possible to attain fully the investigators' original goal: to assess quantitatively four fundamental dimensions constituting the need-for-social-approval matrix. These may now be evaluated individually or in conjunction with each other, in accord with the investigator's purpose.

Because the endorsement of self-descriptive statements can be accounted for largely on the basis of the social desirability value of the item (see Millham \& Jacobson, 1978), it becomes most important to identify, measure, and "operationalize" each of the parameters that contribute to this construct. It is expected that SDI-II will prove a useful tool by which investigators can measure and quantify more precisely the factors that control item endorsement.

\section{REFERENCES}

Ariza, M. J. Self-deception: Unrealistic consistency? Unpublished doctoral dissertation, University of Miami, 1982.

Bentler, P. M., Jackson, D. N., \& Messick, S. Identification of content and style: A two-dimensional interpretation of acquiescence. Psychological Bulletin, 1971, 76, 186-204.

Jacobson, L. I., Kellogg, R. W., Cauce, A. M., \& Slavin, R. S. A multidimensional social desirability inventory. Bulletin of the Psychonomic Society, 1977, 9, 109-110.

Millham, J., \& Jacobson, L. I. The need for approval. In H. London \& J. Exner, Jr. (Eds.), Dimensions of personality. New York: Wiley, 1978.

Millham, J., \& Kellogg, R. W. Need for social approval. Impression management or self-deception? Journal of Research in Personality, 1980, 14, 445-457.

Ramanaiah, N. V., Schill, T., \& Leung, L. S. The test of the hypothesis about the two-dimensional nature of the MarloweCrowne Social Desirability Scale. Journal of Research in Personality, 1977, 11, 251-259.

(Manuscript received for publication June 3, 1983.) 